\title{
Computerised axial tomography and acute neurological problems of childhood
}

\author{
R. E. DAY, J. L. G. THOMSON, AND W. H. SCHUTT \\ From Frenchay Hospital, Bristol
}

SUMMARY The results of computerised axial tomography (CAT) in 80 children with neurological symptoms and/or signs of less than 3 months' duration are discussed in relation firstly to intracranial pathology and secondly to clinical presentation. 26 children had intracranial space-occupying lesions (tumour, abscess, haemorrhage, infarct). CAT was abnormal in 25 of these and diagnostic in 18. A further 20 children had meningitis or encephalitis, and CAT was abnormal in 12. In contrast with this high rate of scans showing pathology, CAT was abnormal in only 4 of the remaining 34 children who had less definite or no intracranial disease.

Analysis of clinical presentation showed that 42 of 69 children presented with persisting neurological signs and of these, 25 had an intracranial space-occupying lesion and 29 had abnormal CAT. Only 5 of 27 children who had symptoms alone or signs lasting less than 24 hours had abnormal $\mathrm{CAT}$, and no intracranial lesion requiring specific treatment was missed. CAT is useful for demonstrating the site, size, and nature of many lesions. The scan may not initially be abnormal in brain stem gliomas and in small subdural collections of fluid.

Computerised axial tomography (CAT) is valuable in diagnosing intracranial pathology, and often demonstrates the nature and extent of a lesion. Clinically it is equally useful for its ability to exclude certain conditions. CAT is also used to monitor hydrocephalus and tumours. In some conditions it may give more information than investigations previously available or thought justified (HarwoodNash and Breckbill, 1976; Baker, 1976). CAT is noninvasive, easy to perform, and does not require admission to hospital. The clinical situations in which it is useful in childhood have not yet been defined. This paper studies its value in a group of 80 children presenting with acute neurological symptoms or signs.

\section{Method}

All children aged 0-15 years who had CAT in the 2year period 1974-1976 at Frenchay Hospital, Bristol, were studied.

\section{Results}

465 children had 632 CAT examinations. These have been divided into groups as shown in Table 1.83

Received 13 May 1977
Table 1 No. of children having CAT in 2 years

\begin{tabular}{lrc}
\hline & No. of patients & No. of CAT scans \\
\hline Hydrocephalus or macrocephaly & 152 & 248 \\
Chronic neurological disoraers & 153 & 166 \\
Acute neurological disorders & 83 & 113 \\
Head injury & 47 & 62 \\
Other & 30 & 43 \\
Total & 465 & 632 \\
\hline
\end{tabular}

patients presenting with symptoms and/or signs of less than 3 months' duration were analysed and children with hydrocephalus or head injury excluded. 2 could not be traced and CAT was unsatisfactory in 1. The remaining 80 patients were divided according to the final diagnosis (Table 2) as well as on the basis of clinical presentation (Tables 3 and 4). We discuss the value of CAT in various pathological conditions in part I of this paper and its value in relation to clinical situations in part II.

\section{PART I CAT FINDINGS RELATED TO DIAGNOSIS}

(A) Tumours. 13 children with primary intracranial tumours (Table 5) and 1 with a secondary meningeal tumour were found. 3 had supratentorial tumours and in each of these CAT was diagnostic and no further investigation was performed before surgery. There was enhancement with Conray in each case 
Table 2 Diagnosis in children presenting with acute neurological disorders

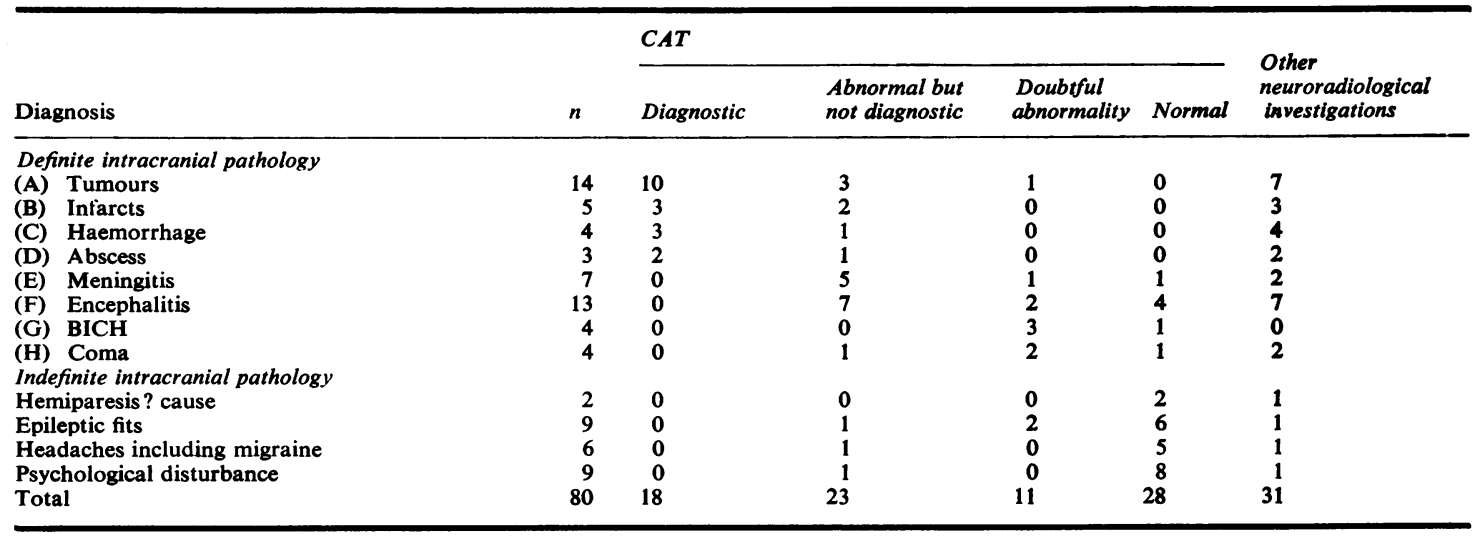

$\mathbf{B I C H}=$ benign intracranial hypertension.

Table 3 Patients with no abnormal neurological signs for more than 24 hours

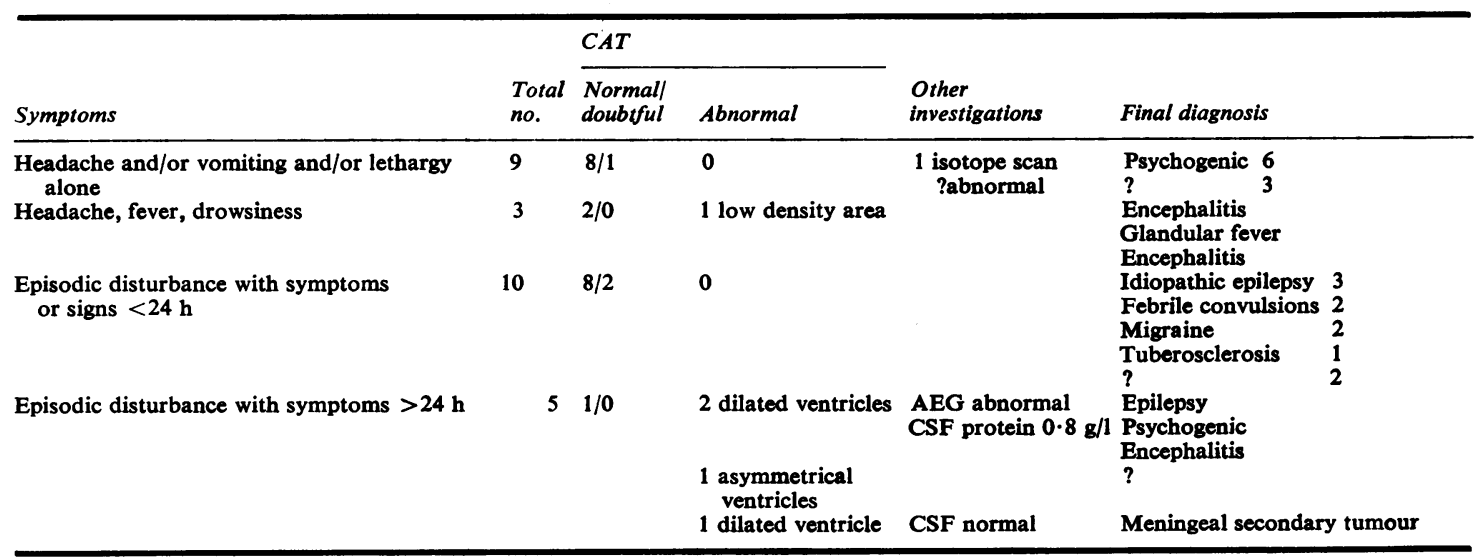

AEG = air encephalogram.

Table 4 CAT findings in patients with abnormal neurological signs

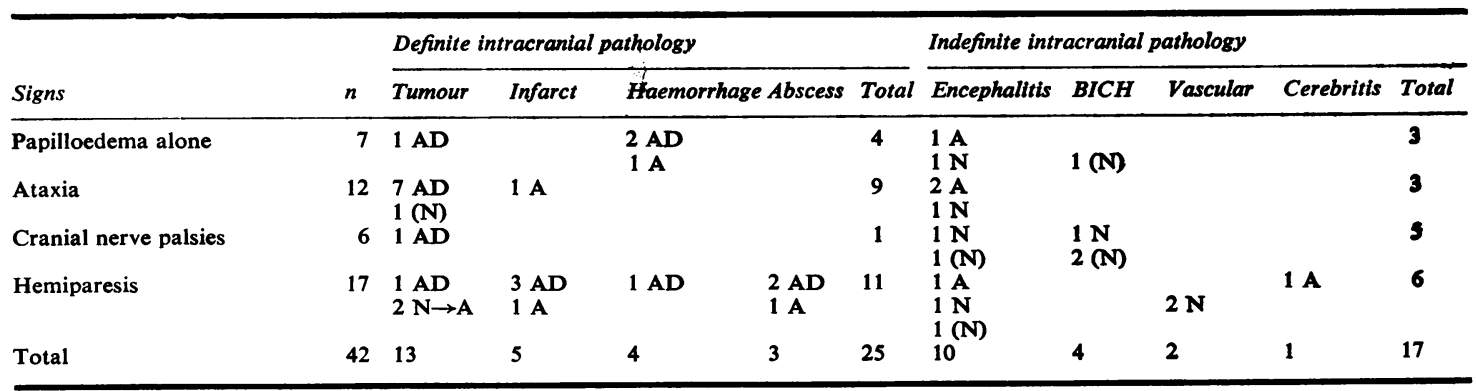

$\mathrm{N}=$ normal; $(\mathrm{N})=$ doubtful abnormality; $A=$ abnormal; $A D=$ diagnostic.

and the cystic nature of the tumour was shown in 2 . 6 children had cerebellar tumours and in each of these CAT was diagnostic of a malignancy; however n 4 of them further investigation was performed before surgery (Table 5). CAT also demonstrated the associated lateral and third ventricular dilatation. There was enhancement with Conray in 5 patients (Figs. 1, 2). 
Table 5 Primary intracranial tumours

\begin{tabular}{|c|c|c|c|c|c|}
\hline \multirow[b]{2}{*}{ Type of tumour } & \multirow[b]{2}{*}{$n$} & \multicolumn{3}{|l|}{$C A T$ findings } & \multirow[b]{2}{*}{$\begin{array}{l}\text { Other } \\
\text { investigations }\end{array}$} \\
\hline & & $\begin{array}{l}\text { Lateral } \\
\text { ventricles }\end{array}$ & $\begin{array}{l}\text { Density of } \\
\text { mass }\end{array}$ & $\begin{array}{l}\text { Enhanced } \\
\text { by Conrav }\end{array}$ & \\
\hline Cerebellar medulloblastoma & 4 & Dilated & High & $\begin{array}{l}3 \text { yes }(1 \text { not } \\
\text { given) }\end{array}$ & $\begin{array}{l}1 \text { RCA } \\
1 \text { Myodil ventriculography }\end{array}$ \\
\hline ependymoma & 1 & Dilated & High & Yes & Myodil ventriculography \\
\hline " astrocytoma & $i$ & Dilated & Cystic & Yes & Myodil ventriculography \\
\hline Cystic astrocytoma pons & $i$ & Dilated & Low & Yes & No \\
\hline \multirow[t]{3}{*}{ Brain stem glioma } & 3 & Normal & Low & No & AEG \\
\hline & & Slight dilatation & Low & No & Myodil ventriculography \\
\hline & & Normal & Isodense & Slight & AEG \\
\hline \multirow{3}{*}{$\begin{array}{l}\text { Ependymoma cerebral hemisphere } \\
\text { Glioma basal ganglia } \\
\text { Glioma cerebral hemisphere }\end{array}$} & 1 & Displaced & Cystic & Yes & No \\
\hline & 1 & Displaced & High & Yes & No \\
\hline & $i$ & Displaced & Cystic & Yes & No \\
\hline
\end{tabular}

RCA $=$ Right carotid arteriogram.

The 4 brain stem tumours were more difficult to diagnose. One child had a cystic astrocytoma of the pons clearly seen on CAT 6 weeks after the onset of symptoms. The second child, who had CAT 3 weeks after the onset of symptoms, showed a little uptake of Conray in the brain stem, which was still considered to be within normal limits at the time (Fig. 3). However, air encephalogram (AEG) 10 days later showed displacement of the aqueduct and 4th ventricle consistent with a brain stem tumour. The third and fourth boys each presented with a hemiparesis and cranial nerve palsies. CAT performed 10 days and 3 weeks, respectively, after onset of symptoms was normal in each case. Repeat CAT 4 weeks later, however, showed a small low density area in the brain stem and investigation with AEG and myodil ventriculography, respectively, confirmed the brain stem swelling and showed posterior displacement of the aqueduct and 4th ventricle, consistent with the diagnosis of brain stem glioma. Diagnosis in the third boy was confirmed at necropsy. The child with meningeal tumour deposits secondary to an undifferentiated tumour in his neck showed mild dilatation of the ventricles consistent with a meningitis tut the meningeal deposits shown at necropsy 18 days later were not seen on the scan.

(B) Infarcts. 5 children had cerebral infarcts. In 3 these were in the territory of the middle cerebral artery and 2 showed the classical wedge-shaped low density area (Fig. 4). The third child with congenital heart disease showed bilateral low density areas with dilated ventricles 6 weeks after the first episode of arterial occlusion. The fourth and fifth children had infarcts in the territory of the anterior cerebral artery. One showed areas of mixed density and the possibility of a tumour was disproved histologically. The high density was presumably haemorrhage. The

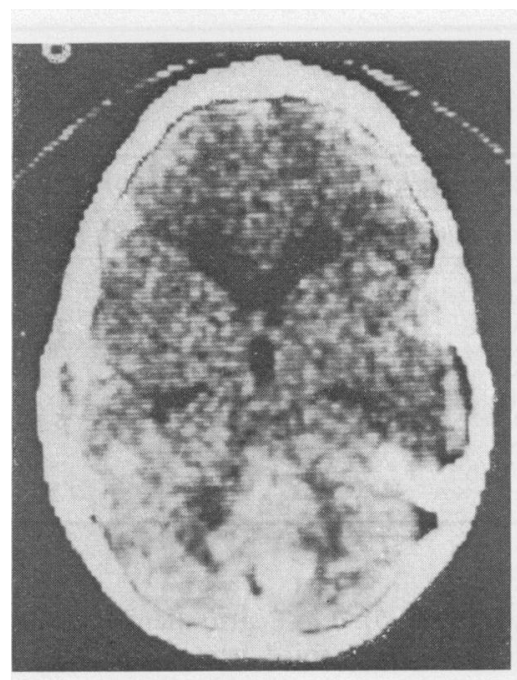

(a)

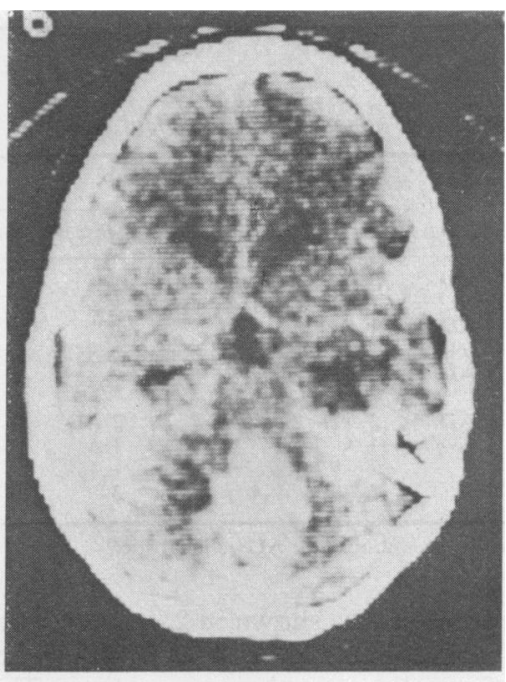

(b)
Fig. 1 Medulloblastoma. (a) High density mass in the posterior fossa. $(b)$ Highlighting with Conray. Note associated dilatation of lateral ventricles. 


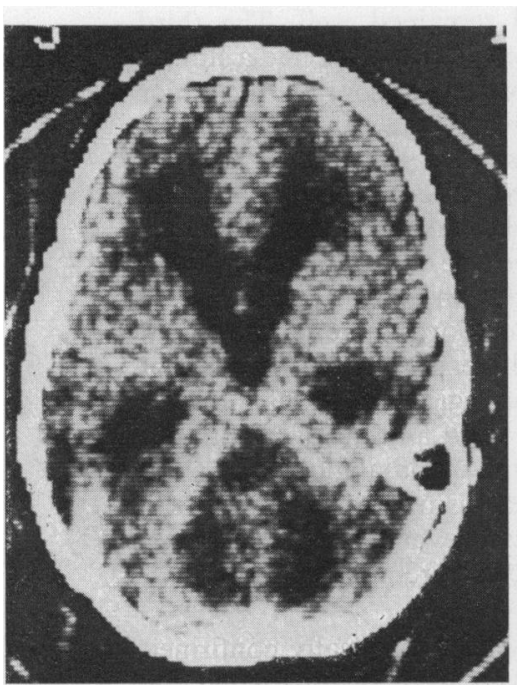

(a)

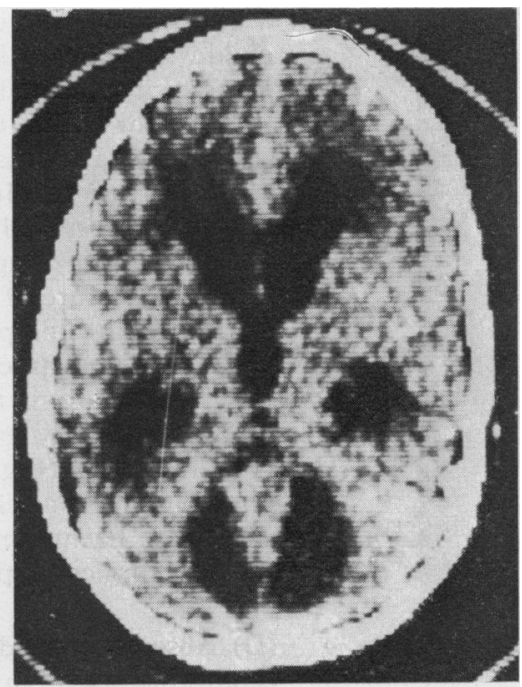

(b)
Fig 2 Cerebellar astrocytoma. (a) Large cystic lesion in posterior fossa with central mass. (b) Enhanced by Conray. fifth child showed the expected dilatation of the ventricle on the side of the lesion as the scan was done 3 weeks after the onset of symptoms. Conray given in the fourth and fifth cases showed some enhancement in the latter. This is known to occur in infarcts particularly if the scan is delayed for 1 week after onset of symptoms, and may cause confusion with tumours if the lesion is not the classical wedge shape. Repeat CAT will however differentiate one from the other. The scan is more accurate than angiography in showing the extent of infarction but will not show the underlying vascular obstruction. Carotid angiography was therefore performed in 2 patients and isotope brain scan in one, all with negative results.

(C) Intracranial haemorrhage. This was seen with and without arteriovenous malformations in 4 children. 3 presented with a history of headaches, vomiting, and papilloedema without lateralising signs. One with an 8-day history of headache was found to have a high density area over the right frontal region con-

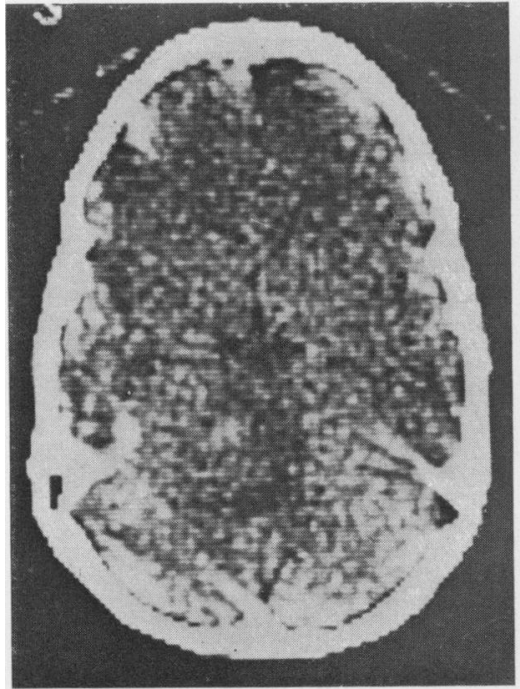

(a)

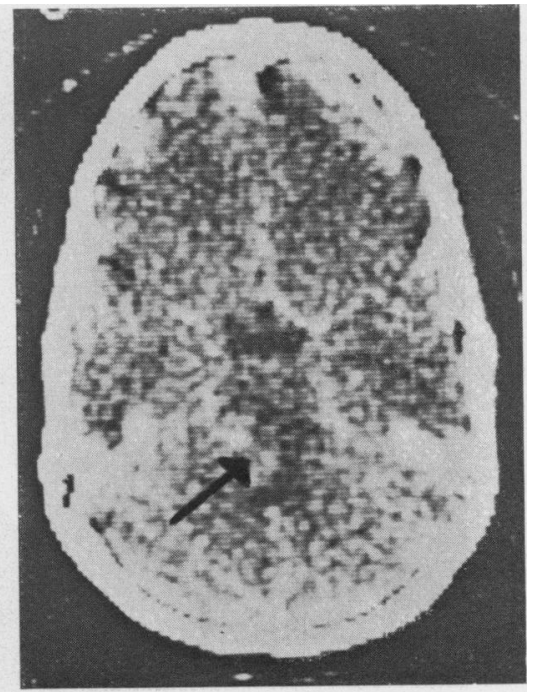

(b)
Fig. 3 Brain stem glioma. (a) Note the mixed high and low density area in front of the fourth ventricle which in (b) shows enhancement after Conray. 


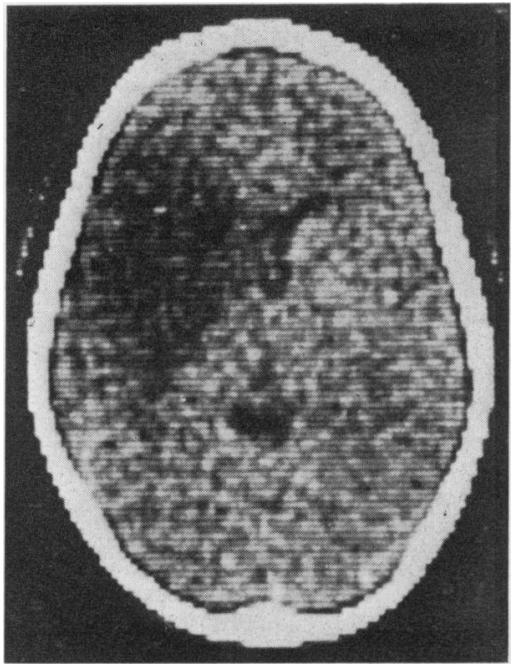

Fig. 4 Cerebral infarct. Low density area in territory of the left middle cerebral artery. Note associated displacement of the lateral ventricles to the right with compression of the left lateral ventricle.

sistent with a subdural haematoma confirmed by carotid angiography and surgery. No cause was found.

The second child with a 3-4 week history was shown to have a low density area highlighting with Conray in the occipital region. This was thought to be a vascular lesion but angiography showed no vascular anomaly. At surgery an old haematoma was aspirated and the child remained well. The third child with a 3-week history of headache had a similar low density area in the occipital region with circular highlighting after Conray (Fig. 5). Angiography showed an angiomatous malformation and led to successful surgery. It was particularly gratifying that in these children without lateralising signs CAT localised the lesion, suggested the diagnosis, and determined the nature of further investigation.

The fourth child presented with a hemiparesis and CAT showed a large high density mass diagnostic of a fresh haematoma in the region of the thalamus. It extended laterally to the vault and medially into the lateral ventricle (Fig. 6a). Carotid angiography (Fig. 6b) showed the arteriovenous malformation but the site of the haematoma as shown on CAT indicated the best surgical approach for removal.

(D) Abscess. 3 children had a surgically confirmed cerebral abscess. In 2 who were being treated for meningitis the scan showed displacement of the ventricles and a large low density area. One child had an angiogram and an isotope brain scan which were both abnormal. The other child was operated on without further investigation.

The third child with sinusitis and a hemiparesis showed low density areas in the right frontal and left parietal regions, with ventricles displaced to the left. This was thought to be consistent with a right frontal lobe abscess. Carotid angiography however suggested a right parasagittal subdural collection of pus with cerebral swelling, which was confirmed at surgery. In retrospect the collection of pus was shown

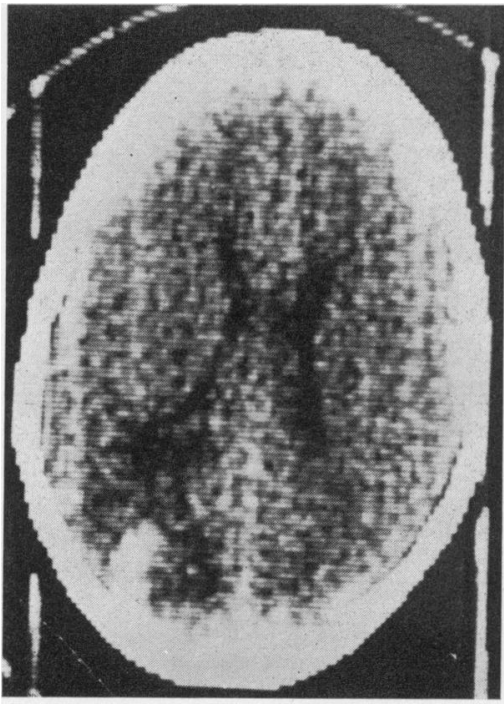

(a)

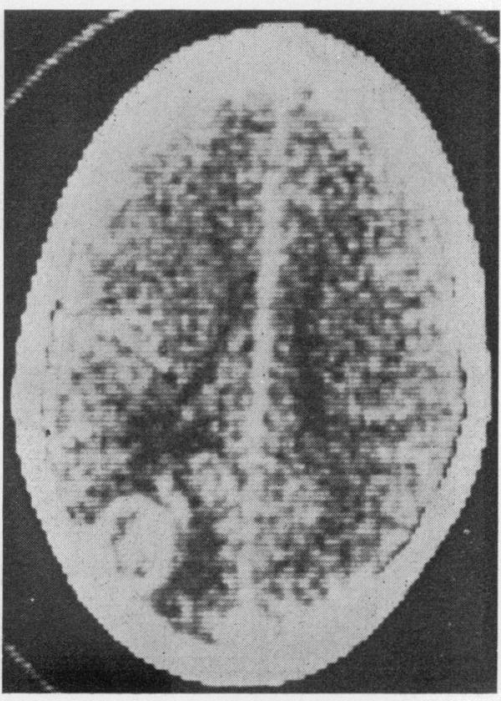

(b)
Fig. 5 Arteriovenous malformation. (a) Low and high density area in the left occipital region with (b) circular highlighting after Conray. Confirmed by arteriography. 


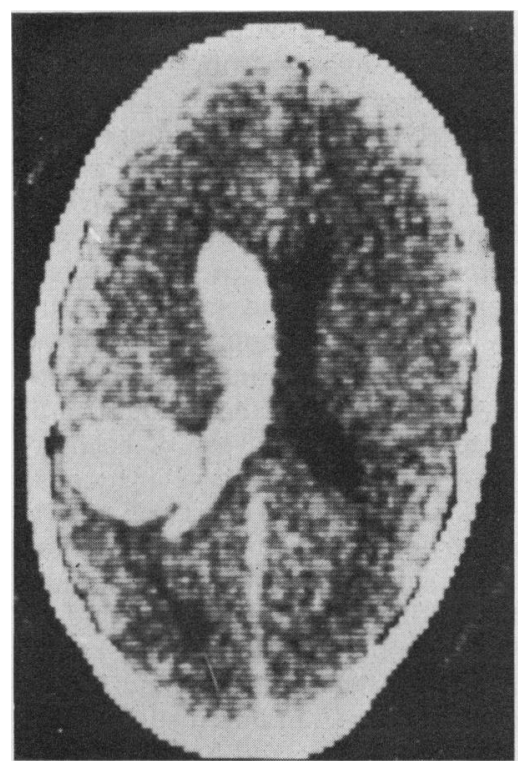

(a)

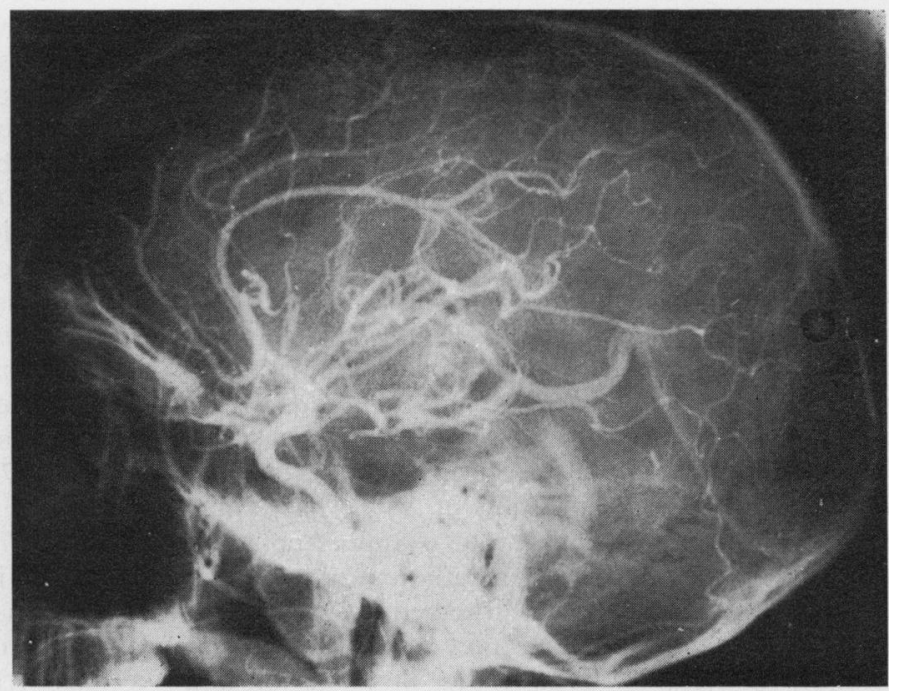

(b)

Fig. 6 (a) Large haematoma (high density) in the region of the left thalamus extending laterally to the vault and medially into the ventricles. (b) Left carotid angiogram showing arteriovenous malformation but not showing as clearly as CAT the site and extent of the haematoma.

in the scan, but the appearances were not correctly interpreted. It should be remembered that both blood and pus in some instances may be isodense with brain substance and thus difficult to see. In the case of intracerebral abscesses, particularly after Conray when a typical thin white ring shadow is produced, the delineation of the lesion is more accurately shown than by other techniques.

(E) Meningitis. This was known to be present in 7 children and CAT was performed because of suspected complications. The aetiology of the meningitis was viral 1 , tuberculous 1 , other bacteria 3 , leukaemic 2. The reason for performing CAT was persistent papilloedema in 4 , continued fever, lethargy, and neck stiffness in 1, convulsions and coma in the child with tuberculous meningitis, and facial palsy in the child with viral meningitis. CAT was definitely abnormal in 5, with dilatation of the ventricles and/ or excess fluid over the cerebral cortex. Abscess was excluded in all cases.

(F) Encephalitis. This was a very mixed group clinically as a definite diagnosis was difficult to make. However of 13 patients, 7 had definitely abnormal scans. In 4 children low density areas were seen and in 2 ventricular dilatation, while 1 had asymmetrical ventricular compression. Of the patients with low density areas, 3 had further neuroradiological investigation. In 1 an isotope scan was normal but in the other 2 isotope scanning and angiography confirmed the CAT findings. One child had bitemporal low density areas suggestive of herpes simplex infection, but was unconfirmed virologically. The low density areas demonstrated on CAT indicated areas of infection and oedema which can usually be distinguished from abscesses, infarcts, and tumours by the absence of displacement. Furthermore abscesses and tumours usually highlight with Conray whereas encephalitis does not. Only 2 of our patients with abnormal scans had Conray and neither showed enhancement.

(G) Benign intracranial hypertension (BICH). This is a poorly defined clinical entity comprising symptoms of raised intracranial pressure with papilloedema in the absence of clinical evidence of intracranial spaceoccupying lesion. It usually remits spontaneously. There were 4 children in this group and 3 had doubtfully abnormal scans while the fourth was normal. One showed low density areas in the right posterior parietal and temporal regions, suggesting possible encephalitis or venous infarction associated with middle-ear disease. The main value of CAT in this condition is in excluding other pathology such as a posterior fossa tumour, which before CAT would have necessitated an AEG or air ventriculography. 
(H) Coma. 4 patients presented with coma for various reasons. One child with Down's syndrome in heart failure was shown clinically to have raised intracranial pressure. CAT showed dilated ventricles and excess cortical fluid. The other 3 had normal scans and were in coma because of carbon dioxide retention, diabetic ketoacidosis, and dehydration.

\section{Discussion}

Our results show that CAT is frequently diagnostic in children with tumours $(10 / 14)$, abscess $(2 / 3)$, intracerebral haemorrhage (3/4), and infarcts $(3 / 5)$. There were only three false-negative examinations (brain stem tumours), two of which later became positive. Thus there is an overall detection rate of these conditions at initial investigation of $88 \%(77 \%$ diagnostic), rising to $96 \%$ if repeat examination is included. $64 \%$ had further neuroradiological investigations, this being a further requirement in intracranial haemorrhage to define the source of bleeding $(12 \%)$. $32 \%$ had surgery without further investigation and with more experience this proportion should increase. There was one false-positive scan (cerebritis/ cerebral abscess) which would almost certainly have been correctly diagnosed had Conray been given.

CAT was diagnostic in all cerebellar and cerebral hemisphere tumours but initially was diagnostic in only 1 out of 4 brain stem tumours. Berger et al. (1976) reviewing CAT in 44 intracranial tumours in children also found CAT diagnostic in all cerebellar tumours, but equivocal in 1 of their 2 brain stem tumours. They concluded that pneumoencephalography is still the procedure of choice in suspected brain stem tumours, but pointed out that CAT may be helpful in defining a cystic component as shown in one of our cases. We feel that as an initial investigation of suspected tumour CAT is very valuable as it demonstrated accurately the size, extent, and content of the tumour as well as any associated ventricular dilatation and will otherwise indicate the next diagnostic procedure required.

Cerebral infarcts are an evolving condition and the CAT appearance depends on the time at which it is performed. CAT in the early stages clearly shows the extent and size of infarction, the presence of haemorrhage or any mass effect which may be surprisingly extensive. Subsequently it will show the amount of resolution with atrophy or cyst formation. Davis et al. (1975) describe the various CAT appearances of infarcts and conclude that angiography may be valuable early to determine the cause of infarction but that later CAT is more informative than angiography or radionuclear scan. Angiography in 2 of our patients performed 2 days and 3 weeks after onset was unhelpful. We conclude that the clinical history and the use of Conray with repeat CAT if necessary will distinguish infarcts from other causes of brain swelling.

CAT is particularly valuable in intracerebral haemorrhage. Scott et al. (1974), comparing angiography and CAT, concluded that CAT provided considerable information about the localisation and extent of the haematoma, the presence of cerebral oedema, intraventricular clot, and hydrocephalus. Furthermore, CAT is the only noninvasive method now available to differentiate an intracerebral clot from an infarct, which has relevance should anticoagulation be considered. In the case of haemorrhage, arteriography is required to show aneurysms which if small will not be shown on CAT (Davis et al., 1976), and to identify feeding and draining vessels of arteriovenous malformations (Pressman et al., 1975). When followed-up over a period of time, an intracranial collection of blood changes from high to low density (New and Aronow, 1976), as shown in 2 of our cases. At an intermediate stage a haemorrhage may be isodense and it may be difficult to define the lesion in subdural haematoma (Davis et al., 1976).

Cerebral infection is a condition which evolves from an area of oedema and cellular infiltration, often progressing to an encapsulated collection of pus. Zimmerman et al. (1976) describe 2 cases similar to ours in which initial CAT showed a low density area they described as cerebritis. Follow-up scans showed the localisation of an intracerebral and subdural abscess cavity. We have found that subdural collections of pus may be difficult to detect initially on CAT as the density is often the same as adjacent normal brain. Consequently, persistent suspicion of subdural abscess in the presence of a doubtful scan indicates the need for further investigation with electroencephalogram, angiography, and even burr holes. On the other hand, we have found that intracerebral abscesses can be reliably detected on CAT particularly after Conray.

The value of CAT in meningitis is to show complications, and we agree with Zimmerman et al. (1976) that CAT is useful in detecting associated infarcts, abscesses, and hydrocephalus. CAT is not generally diagnostic in encephalitis, though we have found patchy areas of low density of inconstant distribution in several cases of uncertain aetiology. The characteristic appearance of low density areas in both temporal regions has been described in herpes simplex encephalitis (Thomson, 1976) and one of our cases showed this distribution. Other virus infections possibly produce a similar appearance (Heathfield et al., 1967). CAT makes it possible to apply brain biopsy accurately to the affected area for virological confirmation. 
PART II CAT FINDINGS RELATED TO CLINICAL PRESENTATION

It is important to determine in which group of patients CAT has most diagnostic value. The patients are divided into two major groups: (1) those with neurological symptoms alone or with signs lasting no longer than 24 hours (27 patients); (2) those with neurological signs for more than 24 hours (42 patients). The children with meningitis and coma are not included in this section as the primary diagnosis is made before CAT.

\section{Group 1.}

Patients with symptoms but neurological signs for less than 24 hours (Table 3). 8 of the 9 patients with headache, with or without vomiting, and lethargy had normal scans. In many of these the illness was eventually thought to have a psychological basis. (One scan was doubtfully abnormal but no specific cause for this was found.) 3 were also pyrexial and the final diagnosis was encephalitis. 15 patients had an episodic disturbance, such as convulsions, loss of consciousness, or dizziness. Of 10 with symptoms and signs for less than 24 hours, none had a definitely abnormal CAT. One 6-year-old child with fits and many depigmented skin lesions was thought to have tuberous sclerosis, but CAT showed only a doubtful low density area which was not considered to be diagnostic. 5 patients had fits with persisting symptoms but no signs. One had a normal CAT and was thought to have idiopathic epilepsy. One child had fits and had dilated ventricles and excessive cortical fluid. He had a raised CSF protein and was thought to have encephalitis. A further boy had a single fit and continued to complain of headache and lethargy. Initial CAT showed a slightly large left ventricle confirmed by AEG and repeat CAT showed no change. His symptoms were thought to be psychiatric in origin and were unchanged 6 months later. The boy with meningeal secondary tumour had fits, and CAT showed dilated ventricles, consistent with meningitis.

CAT in these patients excluded other conditions. Only one patient with definite intracranial pathology presented with symptoms but no signs. This was the patient with meningeal secondary tumour in which the scan was abnormal as described. It is therefore questionable whether it is justified to perform CAT in patients without persisting abnormal neurological signs.

\section{Group 2.}

Patients with abnormal neurological signs. Table 4 indicates those patients with an indefinite diagnosis and those with a definite diagnosis often requiring surgical treatment. The presentation may be very similar in these two groups, i.e. papilloedema, hemiplegia, ataxia, and it is for this reason that CAT is potentially most valuable.

Papilloedema. 7 patients presented with papilloedema as the only abnormal sign and of these, 4 had definite intracranial pathology (3 intracranial haemorrhage, 1 cerebral glioma). CAT was diagnostic in 3 and suggestive in 1 . The remaining 3 patients recovered spontaneously. One child with presumed encephalitis showed a low density area in one parietal region which was still present with some ventricular shift at 5 days, but had disappeared on the eleventh day. Isotope brain scan was normal. Of the other 2 children, one diagnosed as encephalitis with abnormal CSF, and the other as BICH had no definite abnormality on CAT. Neither of these children were investigated further and both recovered fully. CAT therefore identified those children requiring surgical treatment and identified the site, nature, and extent of the lesion.

Ataxia. 10 children presented with ataxia and a further 2 were described as having an unsteady gait without a more specific description. 9 had definite intracranial pathology ( 1 infarct, 6 cerebellar and 2 brain stem tumours). In all 6 cerebellar tumours CAT was diagnostic, but one of the brain stem tumours was not shown. The child with the haemorrhagic infarct presented with headache, vomiting, and unsteadiness. CAT was definitely abnormal but not diagnostic. The other 3 children recovered fully and were thought to have had encephalitis or acute benign cerebellar ataxia. In one, CAT showed slightly dilated ventricles and the CSF contained $15 \mathrm{WBC} /$ $\mathrm{mm}^{3}$. In the second, CSF was normal; CAT showed some compression of the left lateral ventricle which persisted 8 days later. The third child had a normal CAT and CSF and was fully recovered within 3 weeks.

Thus, CAT in an ataxic child will show cerebellar tumours. Brain stem tumours may not be identified early and if there are associated cranial nerve signs or the child deteriorates, repeat CAT or other investigation is indicated.

Cranial nerve palsies. 6 children presented in this way, only 1 having definite intracranial pathology eventually identified as a cystic ependymoma in the right cerebral hemisphere. CAT indicated a tumour and surgery was undertaken without further radiology. The other 5 children recovered fully (BICH 3 , 
encephalitis 2) and none had a definitely abnormal CAT. The 3 diagnosed as BICH presented in a very similar way to the child with the ependymoma, with papilloedema and 6th nerve palsy, although the history was shorter in the latter. The 2 children with encephalitis presented with headache, vomiting, drowsiness, and unilateral facial weakness. One child had papilloedema, and 125 lymphocytes $/ \mathrm{mm}^{3}$ in the CSF.

CAT reliably distinguished the patients with nonprogressive conditions. However, if cranial nerve palsies are associated with ataxia or hemiplegia a normal CAT must be interpreted with caution as CAT was initially normal in 3 of the 4 brain stem tumours.

Hemiparesis. 17 children presented with a hemiparesis and CAT was diagnostic in 7 (infarct 3, abscess 2, haemorrhage 1, tumour 1) (Table 4). It was initially abnormal in a further 4 ; a presumptive diagnosis of encephalitis being made in 1 and further investigation leading to the correct diagnosis in 3 (infarct 1 , abscess 1 , cerebritis 1 ). In the remaining 6 patients CAT was initially normal and in 3 of these further investigation was unhelpful. In one, carotid angiography suggested a venous sinus thrombosis. In 2, clinical deterioration led to repeat CAT which suggested a brain stem glioma, confirmed by AEG.

The 3 children mentioned above in whom initial CAT was abnormal but not diagnostic are of interest. Firstly, the child with the infarct had a 3-week history of hemiparesis when CAT was performed. It showed the classical appearance of a late infarct-a low density area with some highlighting with Conray and dilatation of the ventricle. The 2 children with abscess and cerebritis had very similar histories and CAT appearances. One had a hemiparesis in association with sinusitis and is described in the section on abscess. The other had orbital cellulitis and angiography showed a bare area in the same region as the low density area on CAT. Surgery however showed only cerebral oedema. Conray would almost certainly have distinguished these conditions.

Overall, however, CAT is very helpful in association with the clinical findings in children with hemiparesis, being diagnostic in $41 \% .12$ of the 17 patients had further neuroradiological investigation but this was helpful in only 5 (brain stem tumour 2 , abscess 1 , arteriovenous malformation 1 , venous thrombosis 1), unhelpful in 5, and merely confirmed CAT findings in 2.

\section{Discussion}

Children presenting with histories of less than 3 months' duration suggestive of intracranial patho- logy can easily be divided clinically into two groups, those with and without abnormal neurological signs. In the group with no abnormal neurological signs for more than 24 hours are many who would not previously have been investigated neuroradiologically. In none of these patients was unsuspected intracranial pathology found with CAT, and its value lies in the reassurance it gives to clinicians and patients alike. In those with abnormal neurological signs-papilloedema, ataxia, cranial nerve palsies, and hemiparesis-CAT provides a safe, rapid, and relatively nontraumatic way of distinguishing potentially serious intracranial conditions often requiring specific treatment from those with a more self-limiting benign cause.

In the 13 children with papilloedema and/or cranial nerve palsies and no other signs, CAT clearly distinguished those with tumours and haemorrhage (5) from those with encephalitis or BICH (8). Before CAT, the children with no localising signs would have had air ventriculography or bilateral angiography. Now even in those with haemorrhage who still require angiography the side of the lesion is demonstrated by CAT. In those with cerebellar ataxia and no cranial nerve signs, CAT again distinguished those with tumour (6) and infarct (1) from those with self-limiting conditions (3). However, the 2 children with ataxia and cranial nerve signs both had brain stem tumours and, as discussed above, one of these had a normal CAT.

Acute hemiplegia of childhood has many causes (Isler, 1971) and in our 17 cases CAT was abnormal in 13, 7 showing diagnostic appearances. CAT was initially normal in 2 children with cranial nerve signs and hemiplegia who were later found to have brain stem tumours. It has been the practice to perform angiography on children with acute hemiplegia but this procedure is not without risk and may fail to distinguish haemorrhage from infarction. Normal angiography also does not exclude a vascular aetiology as emboli may disappear within hours or days. In 12 of our patients further neuroradiological investigations were performed but this gave additional information in 5 only.

CAT is therefore diagnostically valuable in children presenting with acute neurological problems. Only half of the children with neurological signs required further neuroradiological investigations. In many cases it provides more information than these traditional techniques, in particular about the exact site and size of a lesion. It is certainly much more acceptable to the child, and can indeed save the child being admitted to hospital.

The CAT scans were performed on an EMI brain scanner CT 1000. 
References

Baker, H. L., Jr. (1976). Computed tomography and neuroradiology: a fortunate primary union. American Journal of Roentgenology, Radium Therapy, and Nuclear Medicine, 127, 101-110.

Berger, P. E., Kirks, D. R., Gilday, D. L., Fitz, C. R., and Harwood-Nash, D. C. (1976). Computed tomography in infants and children: intracranial neoplasms. American Journal of Roentgenology, Radium Therapy, and Nuclear Medicine, 127, 129-137.

Davis, K. R., Taveras, J. M., New, P. F. J., Schnur, J. A. and Roberson, G. H. (1975). Cerebral infarction diagnosis by computerized tomography. American Journal of Roentgenology, Radium Therapy, and Nuclear Medicine, 124, 643-660.

Davis, K. R., Taveras, J. M., Roberson, G. H., and Ackerman, R. H. (1976). Some limitations of computed tomography in the diagnosis of neurological diseases. American Journal of Roentgenology, Radium Therapy, and Nuclear Medicine, 127, 111-123.

Harwood-Nash, D. C., and Breckbill, D. L. (1976). Computed tomography in children. Journal of Pediatrics, 89, 343-357.

Heathfield, K. W. G., Pilsworth, R., Wall B. J., and Corsellis, J. A. N. (1967). Coxsackie B5 infections in Essex, 1965 , with particular reference to the nervous system. Quarterly Journal of Medicine, 36, 579-595.
Isler, W. (1971). Acute Hemiplegias and Hemisyndromes in Childhood. Clinics in Developmental Medicine Nos. 41/42? Heinemann, London.

New, P. F. J., and Aronow, S. (1976). Attenuation measure ments of the whole blood and blood fractions in computed tomography. Radiology, 121, 635-640.

Pressman, B. D., Kirkwood, J. R., and Davis, D. O. (1975) Computerized transverse tomography of vascular lesions of the brain. 1. Arterio-venous malformations. Americarb Journal of Roentgenology, Radium Therapy, and Nuclean Medicine, 124, 208-214.

Scott, W. R., New, P. F. J., Davis, K. R., and Schnur, J. A $\vec{P}$ (1974). Computerized axial tomography of intracerebraL and intraventricular hemorrhage. Radiology, 112, 73-80.

Thomson, J. L. G. (1976). The computed axial tomography in acute herpes simplex encephalitis. British Journal of Radiology, 49, 86-87.

Zimmerman, R. A., Patel, S., and Bilaniuk, L. T. (1976) Demonstration of purulent bacterial intracranial infec $\omega$ tions by computed tomography. American Journal of 4 Roentgenology, Radium Therapy, and Nuclear Medicine ${ }^{N}$ 127, 155-165.

Correspondence to Dr W. H. Schutt, Spina Bifida Unit, Frenchay Hospital, Bristol BS16 1LE. 